\title{
Regulation of proliferation and cell cycle by protein regulator of cytokinesis 1 in oral squamous cell carcinoma
}

\author{
Fanglong Wu ${ }^{1}$, Xueke Shi ${ }^{1}$, Rui Zhang ${ }^{2}$, Yuan Tian ${ }^{1}$, Xiangjian Wang ${ }^{1}$, Changlei Wei ${ }^{1}$, Duo Li ${ }^{1}$, Xiaoyu Li ${ }^{3}$, Xiangli Kong ${ }^{3}$,
} Yurong Liư ${ }^{3}$, Weihua Guo ${ }^{4}$, Yiqing Guo ${ }^{1,5}$ and Hongmei Zhou ${ }^{1}$

\begin{abstract}
Protein regulator of cytokinesis 1 (PRC1), a microtubule-associated protein, has emerged as a critical regulator of proliferation and apoptosis, acting predominantly in numerous tumors. However, its function in oral squamous cell carcinoma (OSCC) is still unknown. To establish the roles of PRC1 in OSCC, 95 oral clinical samples (54 OSCC, 24 oral leukoplakia [OLK], and 17 normal oral mucosa) and seven oral cell lines (6 OSCC and 1 normal oral cell lines) were analyzed using a series of molecular and genomic assays both in vivo and in vitro were conducted in this study. Herein, we provide evidence demonstrating that expression of PRC1 closely correlates with the degree of epithelial dysplasia in OLK $(n=24)(p<0.001)$, and the poor differentiation, large tumor volume, lymph node metastasis, and high-clinical stage in OSCC $(n=54)(p<0.05)$, illustrating that PRC1 has a promotive influence on tumor progression in OSCC. Simultaneously, we observed that PRC1 knockdown in OSCC cell lines caused G2/M phase arrest $(p<0.05)$, inhibited cell proliferation in vitro $(p<0.05)$ and tumor growth in vivo $(p<0.001)$. Furthermore, the effects of PRC1 on the regulation of proliferation and cell cycle transition in OSCC samples were mediated by p53. The p53/PRC1/EGFR signaling pathway was found to be implicated in the tumor progression of OSCC. Based on our data, we demonstrate that PRC1 is a key factor in regulating proliferation and the cell cycle, pointing to the potential benefits of PRC1targeted therapies for OSCC.
\end{abstract}

\section{Introduction}

Oral cancer, including oropharyngeal cancer, is, overall, the sixth most common cancer ${ }^{1}$. An estimated 300,400 new cases and 145,400 deaths occur globally from oral cavity cancer each year, and ample evidence indicates that the incidence of oral cancer is rising in many countries ${ }^{2-4}$. Generally, surgical resection, which may or may not be associated with radio and

\footnotetext{
Correspondence: Hongmei Zhou (zhouhm@scu.edu.cn) or Yiqing Guo (guoyiqing0113@163.com) or Weihua Guo (guoweihua943019@163.com) 'State Key Laboratory of Oral Diseases, Department of Oral Medicine, West China Hospital of Stomatology, Sichuan University, 610041 Chengdu, Sichuan, China

${ }^{2}$ Department of Preventive Dentistry, West China Hospital of Stomatology, Sichuan University, 610041 Chengdu, Sichuan, China

Full list of author information is available at the end of the article

These authors contributed equally: Fanglong $\mathrm{Wu}$, Xueke Shi, Rui Zhang
}

chemotherapy are still the gold standard for treatment. However, the 5 -year overall survival is still about $50 \%$ and the prognosis depends principally on early detection and probable therapeutic approaches ${ }^{3}$. For the early staged tumors, the cure rate is an excellent nearly $95 \%$ with a rate of local recurrence rate of less than $5 \%$, while the cure rate drops to $20-35 \%$ for advanced tumors with frequent recurrence and regional lymph node metastasis ${ }^{5-7}$. Thus, to seek less drug toxicity and obtain better clinical efficacy, the novel targets for the improvement of the patients' quality life is increasingly required.

Molecularly targeted therapy has been endorsed with the aim of guiding personalized treatment by modulating discrete molecular functions that are specific to cancer cells and not the non-malignant tissue ${ }^{8}$. To date, 


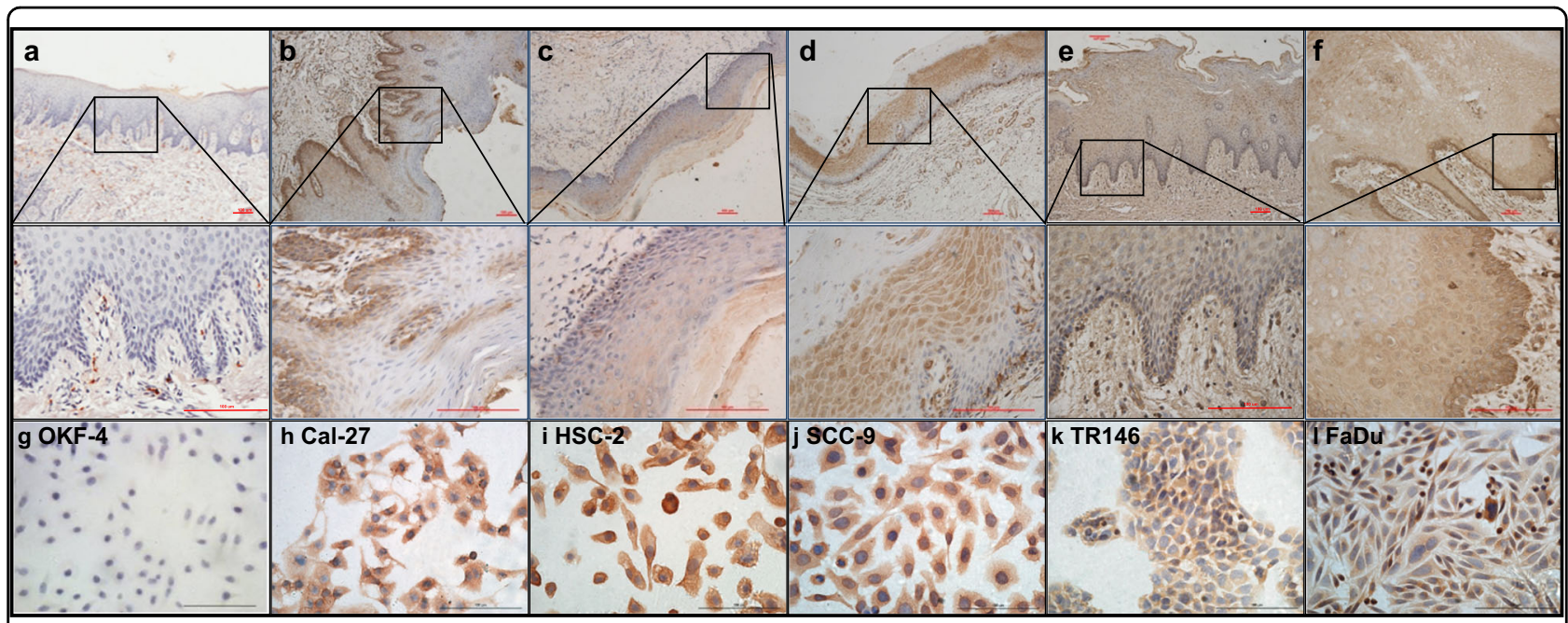

Fig. 1 PRC1 expression is shown in normal epithelium, OLK, and OSCC. a PRC1 was not detected in the normal epithelium. $\mathbf{b}$ In the epithelium of OLK with no dysplasia, IHC demonstrated weak positive expression of PRC1, mainly located in the basal layer cells. c OLK with mild dysplasia exhibited weak positive expression of PRC1, mainly located in the stratum spinosum cells. $\mathbf{d}$ OLK with moderate to severe dysplasia expressed PRC1 in both basal layer and stratum spinosum cells. e Positive expression of PRC1 was observed in highly differentiated OSCC. $\mathbf{f}$ In the presence of poorly differentiated OSCC, diffuse positive and strongly positive cells were observed in epithelium. $\mathbf{g}$ PRC1 expression was negative or weak positive in OKF-4 cells. h-I OSCC cell lines exhibited positive staining commonly observed in OSCC, mainly located in the cytoplasm. Scale bar $=100 \mu \mathrm{m}$

some of the molecularly targeted agents have already been programmed in the clinic with favorable clinical outcomes $^{9,10}$. However, for many molecularly targeted agents, there remains controversy regarding their efficacy and safety. The most controversial aspect of treatment design is the difficultly locating the "Achilles' heel" in thousands of mutant genes and proteins which regulates tumor progression. Using a systems biology approach, the discovery of disease-related genes, proteins, and prognostic biomarkers for cancer treatment can be achieved systematically by studying whole crosstalk among the genes or proteins ${ }^{11-13}$. In our preliminary study, we have successfully developed a systems biology strategy that combines experimental and computational analyses for prediction of epithelial targets in an interactive network between proteins ${ }^{12}$. Based on these, we have forecasted that protein regulator of cytokinesis 1 (PRC1) is one of the potential targets for the treatment of oral cancer ${ }^{12}$.

PRC1, also known as anaphase spindle elongation 1 (ASE1), belongs to the microtubule-associated proteins superfamily ${ }^{14}$. It regulates the proliferation and cytokinesis through crosslinking microtubules, with its crosslinks dynamically tracking antiparallel microtubule overlap ${ }^{15,16}$. So far, given studies have demonstrated that its abnormal expression has a vital role in the proliferation and apoptosis in many tumor types, including breast and bladder cancers ${ }^{17,18}$. Furthermore, its interconnections can also be seen in numerous other proteins such as CCDC69, KIF14, CLASP1, and p53 $3^{19-22}$. However, only Hela and $293 \mathrm{~T}$ cell lines have been studied in this research, with no exploration in head and neck cancer, especially in oral cancer. Accordingly, we speculate that PRC1 might regulate tumor progression in oral squamous cell carcinoma (OSCC). In the present study, we have verified the function and identified potential mechanisms of PRC1 in OSCC through in vivo and in vitro assessments.

\section{Results}

\section{Overexpression of PRC1 in human clinical samples and cell lines}

Firstly, we evaluated the expression of PRC1 in 24 oral leukoplakia (OLK) and 54 OSCC specimens (Fig. 1), which represent different clinicopathologic stages (Tables 1 and 2). In normal oral tissues, the level of PRC1 was almost undetectable or had weakly positive staining (Fig. 1a). However, overexpressed PRC1 in OLK and OSCC was detected (Fig. 1b-f), and the difference was statistically significant when compared to normal oral mucosa $\left(p_{\mathrm{OLK}}=0.000, p_{\mathrm{OSCC}}=0.000\right)$. A comparison of OLK and OSCC demonstrated that PRC1 was expressed in different cellular locations (Fig. 1b-f). In addition, the positive rate and staining score were upregulated in both OLK and OSCC when compared to normal tissue (Fig. S1a, d). Furthermore, the staining score increased with increasing dysplasia in OLK but decreased as differentiation occurred in OSCC while there was no difference in positive rate in the subtypes of OLK or OSCC (Fig. S1b, c, e, f). 
Table 1 The relationship between clinical histopathological features of OLK and expression of PRC1

\begin{tabular}{|c|c|c|c|c|c|}
\hline Factors & Number $(n)$ & Positive rate & Intensity & Staining score & $p$ value \\
\hline \multicolumn{6}{|l|}{ Sex } \\
\hline Male & 14 & $53.43 \pm 13.74$ & $2.21 \pm 0.58$ & $111.93 \pm 13.25$ & \multirow[t]{2}{*}{0.422} \\
\hline Female & 10 & $52.80 \pm 11.08$ & $2.20 \pm 0.42$ & $116.80 \pm 15.89$ & \\
\hline \multicolumn{6}{|l|}{ Age (years) } \\
\hline$\leq 60$ & 16 & $54.31 \pm 13.85$ & $2.19 \pm 0.54$ & $115.19 \pm 15.18$ & \multirow[t]{2}{*}{0.563} \\
\hline$>60$ & 8 & $50.88 \pm 9.45$ & $2.25 \pm 0.46$ & $111.50 \pm 12.91$ & \\
\hline \multicolumn{6}{|l|}{ Location } \\
\hline Buccal & 8 & $53.5 \pm 17.07$ & $2.13 \pm 0.64$ & $110.25 \pm 18.28$ & \multirow[t]{3}{*}{0.627} \\
\hline Tongue & 10 & $51.50 \pm 3.49$ & $2.30 \pm 0.48$ & $114.60 \pm 13.60$ & \\
\hline Others & 6 & $55.50 \pm 8.55$ & $2.17 \pm 0.41$ & $117.83 \pm 9.85$ & \\
\hline \multicolumn{6}{|l|}{ Dysplasia } \\
\hline Without & 5 & $57.60 \pm 19.32$ & $1.80 \pm 0.45$ & $96.80 \pm 4.60$ & \multirow[t]{4}{*}{$0.000^{* * *}$} \\
\hline Mild & 7 & $48.57 \pm 8.83$ & $2.29 \pm 0.49$ & $107.71 \pm 8.28$ & \\
\hline Moderate & 6 & $55.67 \pm 7.66$ & $2.17 \pm 0.41$ & $118.17 \pm 5.81$ & \\
\hline Severe & 6 & $52.33 \pm 14.29$ & $2.50 \pm 0.55$ & $131.33 \pm 8.55$ & \\
\hline
\end{tabular}

Expression of PRC1 in OLK was not relevant to their sex, age, and location of lesions $(p>0.05)$ while exhibited a positive correlation with the degree of epithelial dysplasia $(p=0.000)$. Error bars, mean \pm SD $(n \geq 5) ;{ }^{* * *} p<0.001 ; t$-test or Wilcoxon rank sum test.

To explore the correlation between PRC1 and patients with OLK or OSCC, a method of correlation analysis was introduced to analyze. In this study, we found that the expression of PRC1 in OLK and OSCC was independent of patient sex, age, and location of lesions $(p>0.05)$ while there was a positive correlation with the degree of epithelial dysplasia in OLK $(p=0.000)$ (Table 1$)$, being significantly associated with poor differentiation, large tumor volume, lymph node metastasis and high-clinical stage in OSCC ( $p$ $<0.05$ ) (Table 2). Concomitantly, PRC1 expression as determined using immunostaining in those patients with local lymph node metastasis or at stage T2 was stronger than those at $\mathrm{T} 1$ or having no metastasis $(p<0.05)$. Interestingly, no statistical difference could be found between T2 and T3 $(p=0.499)$, T3 and T4 $(p=0.188)$ (Table 2).

Regarding its behavior in the seven cell lines, as shown in Fig. 1g-l, negative or weakly positive PRC1 staining was observed in OKF-4 cells while the staining in OSCC cell lines, including HSC-2, Cal-27, and SCC-9 (SCC-25 not shown), was positive. Combined with the results of mRNA (Fig. 2b) and protein (Fig. 2c, d), we found that HSC-2 and Cal-27 cells exhibited the highest expression of PRC1 not only at the protein level but also at the mRNA level among the 7 cell lines. Furthermore, HSC-2 cells exhibited the highest expression of "variant 4", whereas Cal-27 cells and the majority of the other OSCC cell lines primarily expressed "variant 1". Accordingly, HSC-2 and Cal-27 cell lines were mainly utilized in the subsequent experiments.
Perturbation of PRC1 changed the biological properties of OSCC cell lines in vitro

To assess the biological changes to OSCC resulting from the perturbation of PRC1, we performed further investigations. Firstly, we established two OSCC cell lines (HSC-2 and Cal-27) with downregulated PRC1. PRC1 si-RNAs (22440-1, 22442-1, and 22443-1) or siNC (CON049) were transfected into cultured HSC-2 and Cal-27 cells respectively under the best infectious condition (ENi.S. $+5 \mu \mathrm{g} / \mathrm{ml}$ Polybrene, multiplicity of infection $=80$ ) resulting in a high transfection efficiency $(83.7 \pm 6.57 \%$ ) (Fig. S2a). Based on RT-PCR data (Fig. 2f, h) and western blot analysis (Fig. 2e, g), we established that PRC1-RNAi (22440-1) was the most effective sequence. Following the decrease in expression of PRC1 in HSC-2 and Cal-27 cells, we further explored their cell biology. Flow cytometry (FCM) was conducted to analyze cell cycle, as shown in Fig. 3c. The cells were observed to be in G2/M phase arrest in the experimental group (si-PRC1) compared to si-NC or blank $(p<0.05)$. Similarly, a comparable situation was observed with Cal-27 cells $(p<0.001)$.

The role of PRC1 on proliferation was then evaluated. Using a CCK-8 analytic approach, as shown in Fig. 3a and $b$, a significantly lower rate of proliferation was observed in si-PRC1-treated cells compared with those transfected with the untargeted-control (si-NC) and blank after $48 \mathrm{~h}$. 
Table 2 The association between clinical histopathological features of OSCC and expression of PRC1

\begin{tabular}{|c|c|c|c|c|c|}
\hline Factors & Number $(n)$ & Positive rate & Intensity & Staining score & $p$ value \\
\hline \multicolumn{6}{|l|}{ Sex } \\
\hline Male & 37 & $82.14 \pm 8.23$ & $2.68 \pm 0.48$ & $217.86 \pm 33.48$ & \multirow[t]{2}{*}{0.881} \\
\hline Female & 17 & $83.59 \pm 7.57$ & $2.64 \pm 0.49$ & $219.41 \pm 34.75$ & \\
\hline \multicolumn{6}{|l|}{ Age (years) } \\
\hline$\leq 60$ & 31 & $83.18 \pm 7.76$ & $2.64 \pm 0.49$ & $218.18 \pm 37.45$ & \multirow[t]{2}{*}{0.930} \\
\hline$>60$ & 23 & $82.55 \pm 8.11$ & $2.68 \pm 0.48$ & $219.09 \pm 30.44$ & \\
\hline \multicolumn{6}{|l|}{ Location } \\
\hline Buccal & 10 & $80.30 \pm 8.59$ & $2.80 \pm 0.42$ & $223.20 \pm 33.62$ & \multirow[t]{5}{*}{0.936} \\
\hline Tongue & 16 & $83.13 \pm 8.19$ & $2.60 \pm 0.51$ & $214.20 \pm 35.46$ & \\
\hline Gingival & 12 & $82.25 \pm 8.00$ & $2.63 \pm 0.52$ & $214.38 \pm 32.94$ & \\
\hline Floor of oral & 12 & $86.25 \pm 7.82$ & $2.63 \pm 0.52$ & $225.13 \pm 35.59$ & \\
\hline Others & 4 & $82.67 \pm 2.08$ & $2.67 \pm 0.58$ & $219.67 \pm 43.04$ & \\
\hline \multicolumn{6}{|l|}{ Differentiation } \\
\hline Well & 31 & $83.74 \pm 7.74$ & $2.44 \pm 0.51$ & $202.41 \pm 32.49$ & \multirow[t]{3}{*}{$0.003^{*}$} \\
\hline Moderate & 16 & $81.73 \pm 7.12$ & $2.87 \pm 0.35$ & $234.07 \pm 27.29$ & \\
\hline Poor & 7 & $80.20 \pm 9.66$ & $3.00 \pm 0.00$ & $240.60 \pm 28.96$ & \\
\hline \multicolumn{6}{|l|}{ T stage } \\
\hline $\mathrm{T} 1$ & 20 & $82.06 \pm 8.21$ & $2.41 \pm 0.51$ & $194.53 \pm 25.52$ & \multirow[t]{4}{*}{$0.003^{*}$} \\
\hline $\mathrm{T} 2$ & 10 & $82.60 \pm 8.10$ & $2.80 \pm 0.42$ & $230.20 \pm 31.84$ & \\
\hline T3 & 8 & $86.46 \pm 5.94$ & $2.55 \pm 0.52$ & $219.364 \pm 39.45$ & \\
\hline $\mathrm{T} 4$ & 16 & $79.56 \pm 7.44$ & $3.00 \pm 0.00$ & $239.67 \pm 22.38$ & \\
\hline \multicolumn{6}{|c|}{ N (lymph node metastasis) } \\
\hline No & 28 & $82.41 \pm 8.29$ & $2.82 \pm 0.40$ & $231.50 \pm 29.26$ & \multirow[t]{2}{*}{$0.004^{*}$} \\
\hline N1N2 & 26 & $83.00 \pm 7.27$ & $2.48 \pm 0.51$ & $203.44 \pm 33.49$ & \\
\hline \multicolumn{6}{|l|}{ Clinical stage } \\
\hline$|/| \mid$ & 33 & $82.19 \pm 7.74$ & $2.55 \pm 0.51$ & $206.71 \pm 31.39$ & \multirow[t]{2}{*}{$0.005^{*}$} \\
\hline III/IV & 21 & $83.75 \pm 7.72$ & $2.81 \pm 0.40$ & $235.69 \pm 32.35$ & \\
\hline
\end{tabular}

No statistical difference between PRC1 and sex, age, tumor location was found in patients with OSCC. However, increased PRC1 was significantly associated with poor differentiation, large tumor volume, lymph node metastasis and high-clinical stage $(p<0.05)$. Error bars, mean \pm SD $(n \geq 4)$; ${ }^{*} p<0.05$; $t$-test or Wilcoxon rank sum test.

To understand whether PRC1 has a direct functional role in the regulation of cell death in OSCC, we performed FCM, trypan blue and propidium iodide (PI)/ calcein fluorescence staining for further exploration. However, our study showed that no statistical difference was observed in the number of apoptotic HSC-2 or Cal-27 cells due to the intervention of si-PRC1 compared to siNC or blank (Fig. 3d). Also, cell death in HSC-2 and Cal27 cells could not be induced by PRC1 knockdown (Fig. S2b-g). Taken together, these data suggest that downregulation of PRC1 induces OSCC to stay in the G2/ $\mathrm{M}$ phase, inhibiting cell proliferation, but has no clear effect on cell death.
Downregulation of PRC1 inhibited tumor growth in vivo

After verifying that the expression behavior of PRC1 was closely related to proliferation and cell cycle in vitro, we continued to explore its effects on tumor growth. Totally, 18 nude mice were utilized in subcutaneous tumorigenicity assay. We found that the subcutaneous scleroma could be detected after inoculation in the si-NC and control groups on the $5^{\text {th }}$ day and after 7-10 days in the si-PRC1 group. As shown in Fig. $4 a-c$, tumors explanted from the si-PRC1-treated group had smaller volumes $\left(468.30 \pm 116.83 \mathrm{~mm}^{3}\right)$ and their weights $(0.3 \pm 0.16 \mathrm{~g})$ were lower than those from the untreated groups (si-NC and blank), differences 


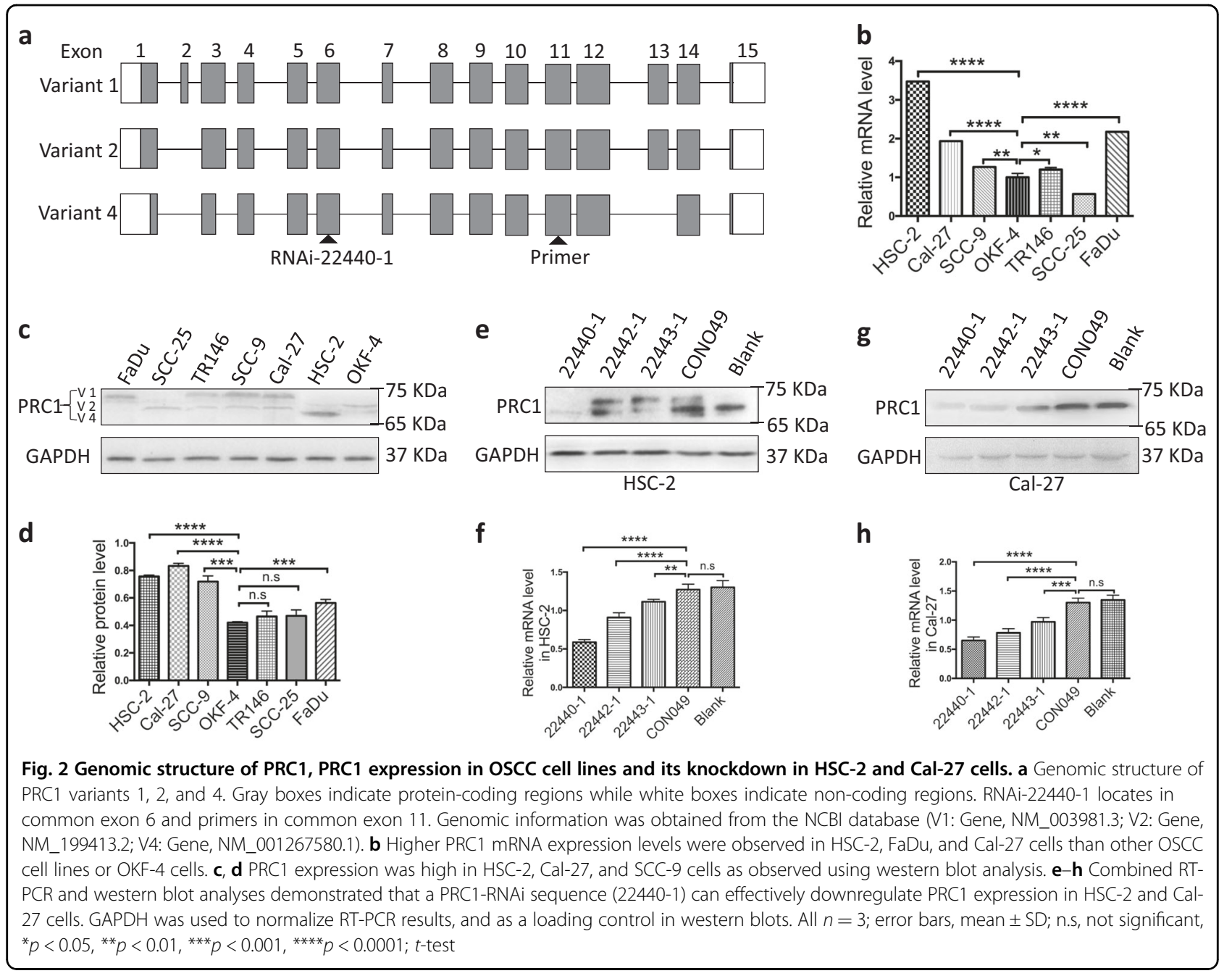

that were statistically significant $(p<0.05)$. As judged by IHC, expression of PRC1 and Ki-67 was reduced significantly in the si-PRC1-perturbed group when compared to the control groups (Fig. 4d, e). Thus, these data indicate that reduced expression of PRC1 inhibits the tumor growth in vivo through suppression of cell proliferation.

\section{The related mechanism of PRC1 in the regulation of proliferation of OSCC}

To understand the potential mechanism of PRC1 in cell proliferation, we measured the expression of epidermal growth factor receptor (EGFR), transforming growth factor $\beta$ type II receptor (T $\beta$ RII) and Aurora kinase A (AURKA), which are closely associated with proliferation and cell cycle, using western blot analysis. The expression of EGFR in HSC-2 cells was weaker than T $\beta$ RII and AURKA in the si-PRC1-perturbed group (Fig. 5a, b, e, f). Unlike in HSC-2 cells, the protein levels of both EGFR and TRRII decreased in Cal-27 cells following PRC1 knockdown, while there was no significant change in AURKA expression compared with the control groups (Fig. 5a, b, e, f), suggesting that decreased EGFR levels following downregulation of PRC1 is a common event in OSCC.

Since PRC1 is a key target of p53, we evaluated the expression of PRC1 in HSC-2 and Cal-27 cells after both activation and inhibition of p53. Following activation by Nutlin-3, expression of both p53 and pho-p53 $\left(\mathrm{Ser}^{392}\right)$ increased significantly in HSC-2 and Cal-27 cells while PRC1 decreased (Fig. 5c, g, h), indicating that p53 negatively affects PRC1 expression. Conversely, expression of PRC1 was increased by the downregulation of p53 in HSC-2 and Cal-27 cells treated with Pifithrin- $\alpha$, an inhibitor of p53 (Fig. 5d, i, j).

\section{Discussion}

In recent years, PRC1, a protein closely related to cytokinesis, has already been well documented for its structure and functions ${ }^{14,16,20,21,23}$. By alternative splicing 


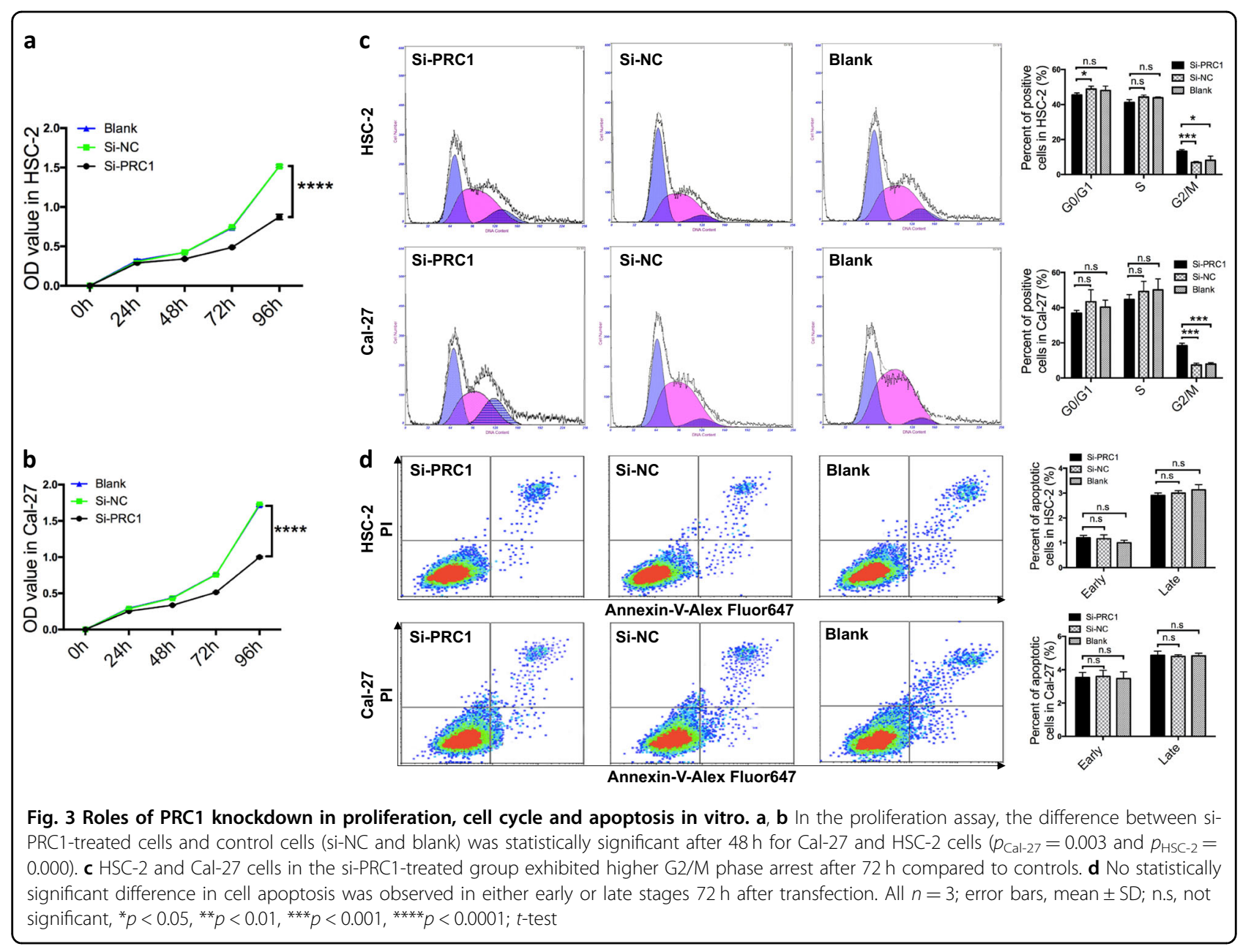

into three variants (variant 1 , variant 2 and variant 4 ), as shown in Fig. 2a and c, it can be considered a substrates of cyclin-dependent kinases, closely associated with the binding of microtubules and assembly of central spindles $^{14,16,23}$. Previous studies have shown that knockdown or knockout of PRC1 is an efficient method of inhibition of tumor growth including breast, bladder and cervical cancers ${ }^{15,18,24,25}$. In this study, we devised a systems biology strategy that progressively cycled experiments and computations to predict and verify the expression of PRC1 in 7 cell lines and 95 clinical samples. Both the positive rate and staining score of PRC1 increased gradually in normal tissue, OLK and OSCC (Fig. S1a, d), indicating that PRC1 has a promoting role in oral tumorigenesis. Combined with the clinical data (Tables 1 and 2), we have provided evidence showing that a close correlation between PRC1 and OSCC progression exists, suggesting that PRC1 has a potential role in predicting malignancy and prognosis of OSCC.

Under normal conditions, presenting circular lines around cell nucleus at interkinesis, PRC1 locates in the chromosomal centromere at prophase then anchors with the mitotic spindle during metaphase and the first half of anaphase, aiming to inhibit elongation of the spindle ${ }^{26}$. It's principle function is to assemble in the central spindle to recruit actin and kinases at telophase ${ }^{26}$. Our study suggests that knockdown PRC1 induces more HSC-2 or Cal27 cells to enter $\mathrm{G} 2 / \mathrm{M}$ phase arrest resulting in the termination of karyomitosis (Fig. 3c). Since PRC1 expresses highly in the $\mathrm{G} 2$ phase (Fig. 3c), it regulates elongation and polarity of the spindle ${ }^{27,28}$. Thus, its effective downregulation blocks the normal process of elongation and polarization of the spindle, causing G2/M phase arrest. These findings also suggest that it is a common event for PRC1's involvement in regulating the cell cycle. The three variants of PRC1 might not have a different role in this process in OSCC cell lines since HSC-2 cells exhibit the highest expression of "variant 4", whereas Cal-27 and the majority of the other OSCC cell lines primarily express "variant 1".

Furthermore, G2/M cell cycle arrest could result in the inhibition of cell proliferation ${ }^{1}$. Previous studies have shown that some cancer cells are highly dependent on PRC1 for proliferation ${ }^{29,30}$. For instance, Shimo et al. ${ }^{18}$ 


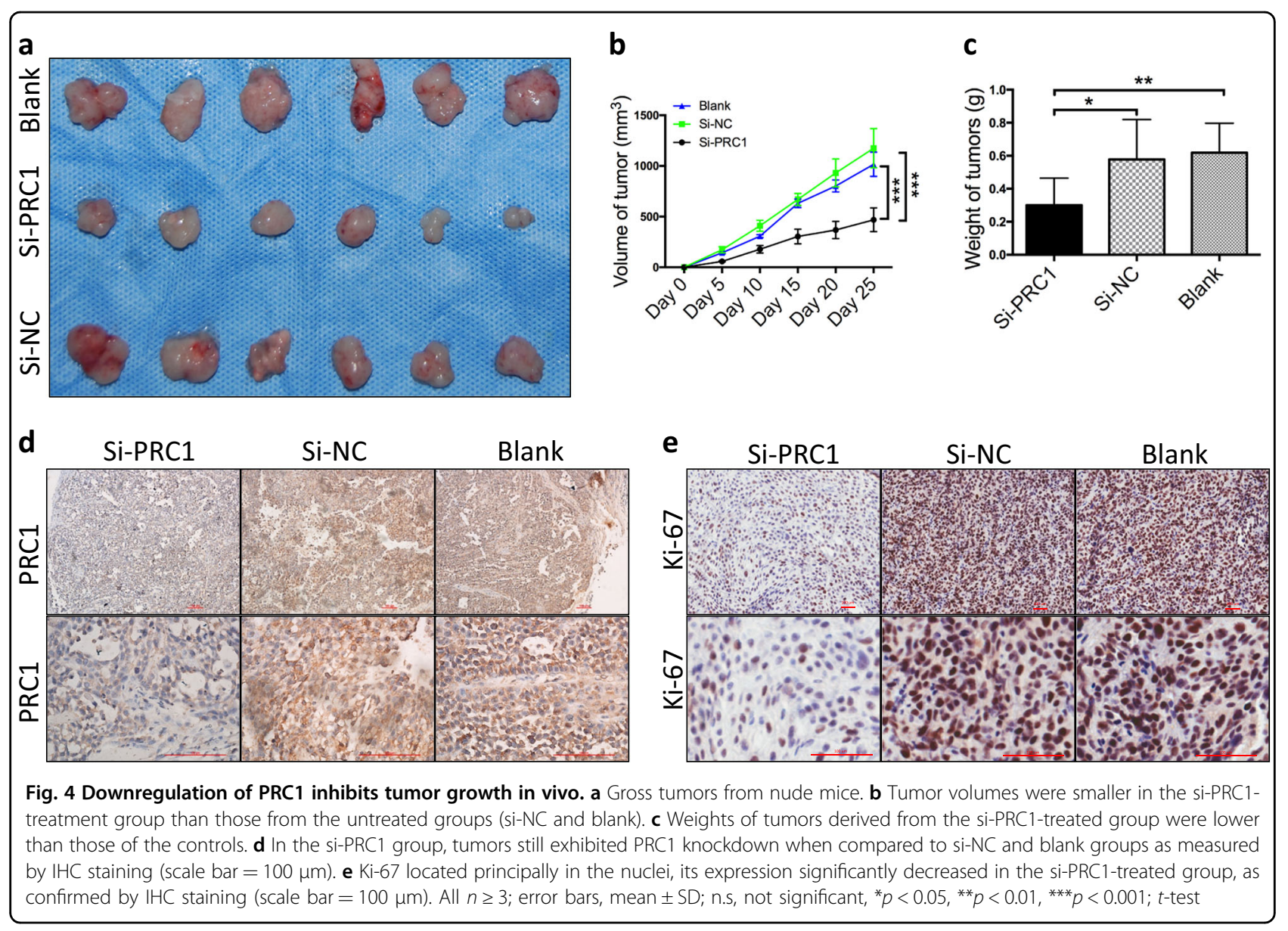

found that intervention with siRNA against PRC1 in breast cancer cells effectively suppressed its expression and inhibited the proliferation of the cells. Similarly, our data also confirms this notion that downregulated PRC1 also inhibits tumor growth without significant toxicity to the cells (Fig. 4a, b, c).

In addition, interference of proliferative signaling is another appealing approach to inducing cell apoptosis in tumor cells since its proliferative response in normal cells while perturbed in most cancers ${ }^{31-33}$. In this study, we additionally performed FCM (Fig. 3d), and evaluated trypan blue and $\mathrm{PI} /$ calcein fluorescence staining (Fig. S2b-g) to investigate if downregulating PRC1 would induce any modification to cell death, including apoptosis in OSCC. However, we did not find that cell death in HSC-2 and Cal-27 cells was induced by PRC1 knockdown. Unlike the findings of Zhang et al. ${ }^{34}$ that PRC1 knockdown in two gastric carcinoma cell lines, AGS and HGC27, induced apoptosis, no significant difference in apoptosis was observed in HSC-2 and Cal27 cells treated with si-PRC1 at prophase or telophase (Fig. 3d), demonstrating that the effect of PRC1 on cell apoptosis is highly tissue and tumor-specific. We propose an alternative hypothesis, that si-PRC1 might inhibit other signaling pathways such as Bcl-2 or Caspase, subsequently leading Cal-27 and HSC-2 cells to become resistant to cell death, including apoptosis, necrosis, and programmed cell death.

In vivo, we found that downregulation of PRC1 also inhibited the proliferation of OSCC which is in good agreement with the in vitro results (Figs. $3 \mathrm{a}, \mathrm{b}$ and $4 \mathrm{a}-\mathrm{c}$ ). Furthermore, expression of PRC1 and Ki-67 decreased significantly in the si-PRC1-treated group compared to the untreated groups (Fig. 4d, e). Ki-67, a proliferating nuclear antigen during cell cycle phases G1, S, G2, and M, is closely related to karyomitosis in the process of proliferation ${ }^{35,36}$. Supporting our results, Dwivedi et al. ${ }^{37}$ also found that increased Ki-67 could be used to assess the severity of epithelial dysplasia and histological grading of OSCC. In sum, downregulation of PRC1 in OSCC could inhibit proliferation, subsequently leading to suppression of tumor growth in vivo.

Mechanistically, EGFR, T $\beta$ RII, and AURKA related to the proliferation and cell cycle were included for the exploratory study ${ }^{38-42}$. The expression of EGFR was found to decrease, corresponding to PRC1 expression 


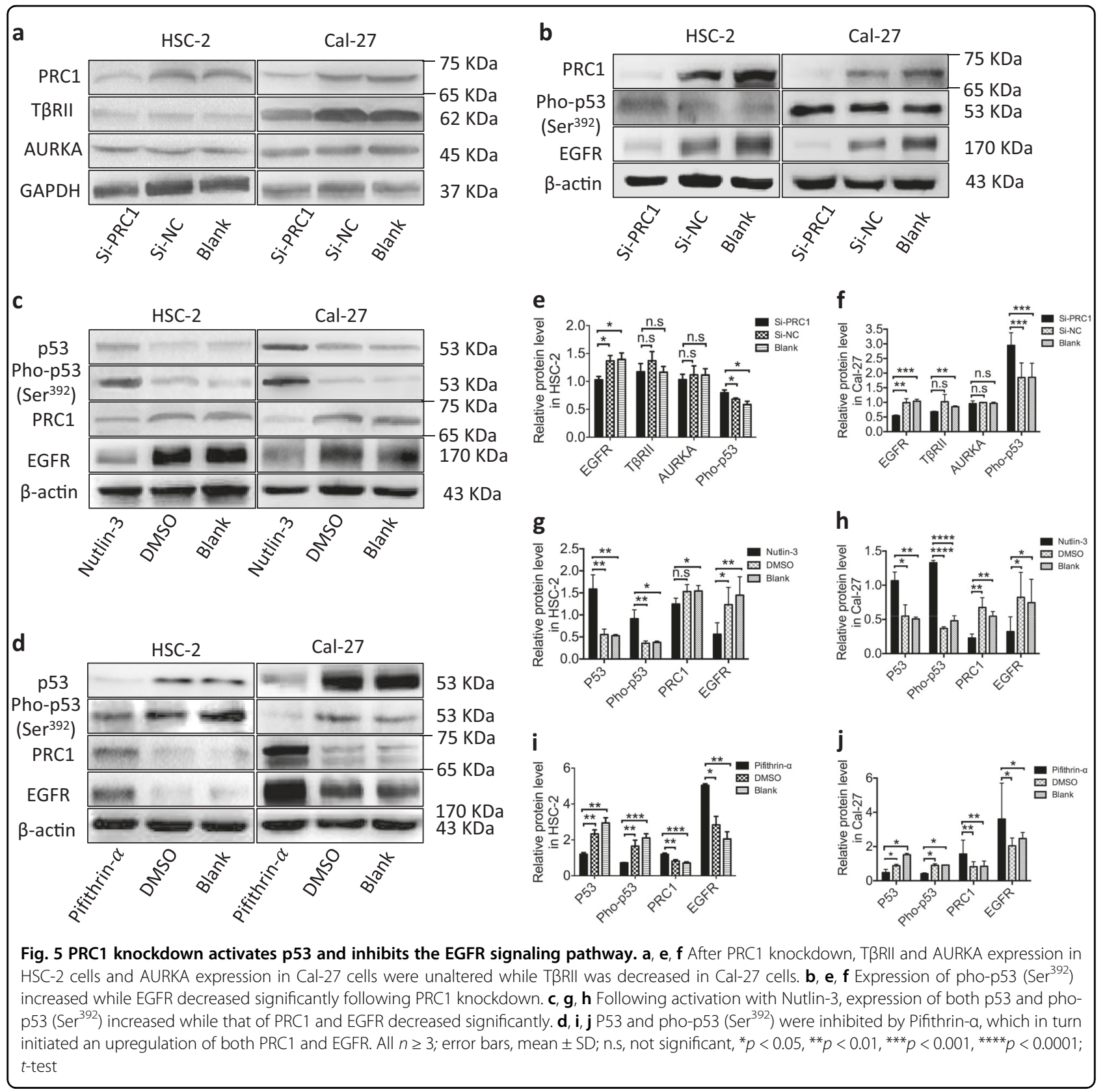

in the si-PRC1-treated group (Fig. 5b, e, f), indicating that PRC1 regulates EGFR signaling, consequently mediating cell proliferation. Since the p53 binding site locates in the EGFR promotor ${ }^{43,44}$, we further detected its expression at the protein level. Intriguingly, pho-p53 $\left(\mathrm{Ser}^{392}\right)$ was attenuated by si-PRC1, suggesting that PRC1 exerts a negative effect on p53 (Fig. 5b, e, f). Furthermore, based on our data (Fig. 5c, g, h), we found that p53 and pho-p53 ( $\mathrm{Ser}^{392}$ ) were both activated by Nutlin-3 which is a p53 activator, thereby leading to the downregulation of PRC1. Conversely, a decrease in p53 and pho-p53 (Ser ${ }^{392}$ ) caused by Pifithrin- $\alpha$ (a p53 inhibitor) augmented PRC1 expression (Fig. 5d, i, j). These results indicate that there is a loop between p53 and PRC1 (Fig. 6). Similarly, Liu et al. ${ }^{21}$ showed that p53 can directly inhibit the transcription of PRC1 with the result of regulating tumor cell mitosis and G2/M phase arrest. In addition, we found that the expression of EGFR changed in line with PRC1 resulting from treatment with Nutlin-3 or Pifithrin- $\alpha$ (Fig. $5 c, d, g-j$ ), further supporting the notion that PRC1 mediates the EGFR signaling pathway. Furthermore, the downstreams of EGFR pathway such as MAPK, JNK, ERK1/2, and so on exerting a regulation of proliferation in 


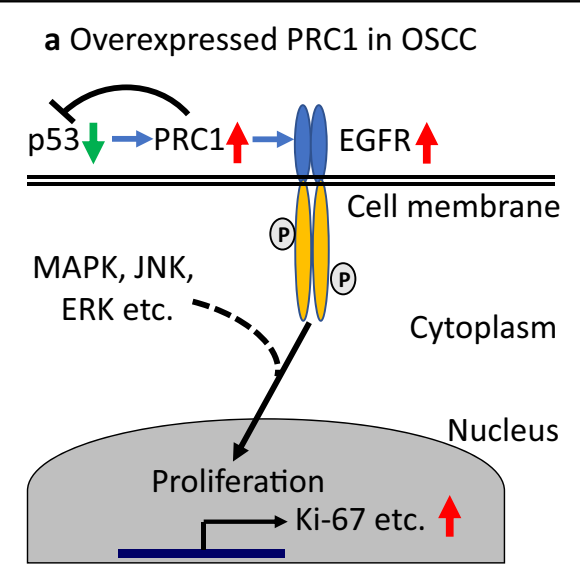

b PRC1 knockdown in OSCC

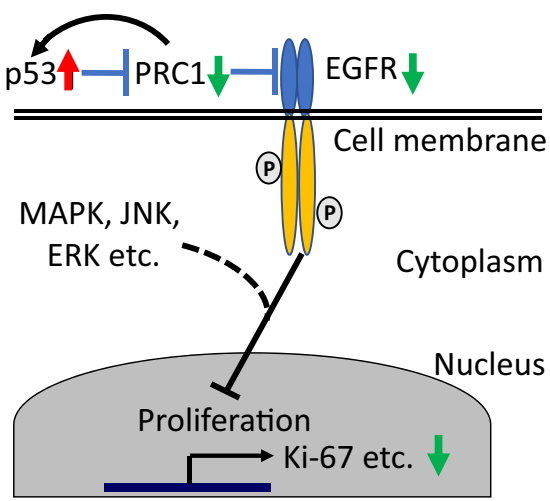

Fig. 6 Schematic overview of p53/PRC1/EGFR in OSCC model. a In OSCC, inhibition of p53 leads to overexpression of PRC1, which in turn further suppresses p53. The EGFR signaling pathway is enhanced by overexpressed PRC1, subsequently leading to the promotion of proliferation. $\mathbf{b}$ After PRC1 knockdown in OSCC, p53 is upregulated which causes additional inhibition of PRC1 as feedback, which consequently suppresses the EGFR pathway and inhibits proliferation

numerous tumors including oral cancer have been well documented $^{45-47}$. In sum, these results suggest that the p53/PRC1/EGFR signaling axis is involved in the process of promoting cell proliferation, causing an abnormity of cell cycle transition in OSCC (Fig. 6).

Unlike the expression of EGFR regulated by PRC1 in HSC-2 and Cal-27 cell lines, we found that TßRII expression decreased in Cal-27 cells with no significant change in HSC-2 cells (Fig. 5a, e, f), illustrating that TßRII might exhibit paradoxical roles either as a suppressor or promotor of PRC1 in OSCC progression. An alternative hypothesis, supported by the variants of PRC1 in HSC-2 and Cal-27 cells, can be proposed, suggesting that variant 4 of PRC1 in HSC-2 cells may not be involved in regulating the expression of TRRII in OSCC. These findings suggest that the functional differences among these variants do exist and so utilization of PRC1 and its variants as an oral cancer therapy should be afforded more attention. With respect to AURKA, unlike the findings of Pan et al. ${ }^{48}$, who reported that it could destruct bipolar spindle structure, suppress tumor growth and induce cell apoptosis in OSCC, no significant change in AURKA expression was observed in our study (Fig. 5a, e, f), indicating it was not mediated by PRC1 in OSCC or other pathways, such as the co-binding of proteins that induces effects to those observed during mitosis.

In summary, based on our findings, for the first time, we have shown elevated expression of PRC1 in human OSCC tissues and an association with OSCC progression. Decreased expression of PRC1 suppresses cell proliferation and causes G2/M phase arrest but has no effect on apoptosis. Importantly, we have provided evidence that the p53/ PRC1/EGFR signaling pathway is involved in the function of PRC1 in cell proliferation and cell cycle transition during tumor development in OSCC, highlighting the potential of PRC1 to become a novel target for OSCC treatment.

\section{Methods and materials}

\section{Patients and tissue samples}

In total, 95 samples from 54 patients with OSCC and 24 with OLK who underwent surgical resection and 17 normal individuals who received craniofacial plastic surgery or tooth extractions were obtained from the West China Hospital of Stomatology in Sichuan University. Tissue samples were immediately fixed in $10 \%$ buffered formalin and then embedded in paraffin prior to sectioning. The presence of cancer in tumor samples was confirmed by pathological examinations. This study was approved by the Human Research Ethics Committee of West China Hospital of Stomatology in Sichuan University (No. WCHSIRB-D-2012-0031-R1).

\section{Cell lines and culture conditions}

A total of seven kinds of cell lines including HSC-2 (American Type Culture Collection [ATCC], Manassas, VA, USA), TR146 (European Collection of Authenticated Cell Cultures [ECACC], Porton Down, Salisbury, UK), SCC-25 (ATCC, Manassas, VA, USA), FaDu (ATCC, Manassas, VA, USA), SCC-9 (ATCC, Manassas, VA, USA), Cal-27 (State Key Laboratory of Oral Disease, Sichuan, China) and OKF-4/TERT-1 (Harvard Skin Disease Research Center, Brigham and Women's Hospital, Boston, MA, USA) were included in this research. The anatomical sites of origin of these cell lines are as follows: oral cavity (HSC-2), tongue (SCC-9, SCC-25 and Cal-27), floor of mouth (OKF-4/TERT-1) and hypopharynx (FaDu and TR146). HSC-2, TR146, $\mathrm{FaDu}$ and Cal-27 cells were maintained in Dulbecco's 
modified Eagle's medium (DMEM) (Gibco, Grand Island, NY, USA). SCC- 9 and SCC- 25 cells were cultured in a 1:1 mixture of DMEM/F-12 medium. OKF-4/ TERT-1 cells were cultivated in keratinocyte or Ham's F-12 culture medium. Each base medium was supplemented with $10 \%$ fetal bovine serum (Gibco, Grand Island, NY, USA). Cells were cultured at $37^{\circ} \mathrm{C}$ in a humidified atmosphere with $5 \% \mathrm{CO}_{2}$.

\section{Immunohistochemistry (IHC) and immunocytochemistry (ICC)}

Human and mouse tumors were dissected and immediately fixed in $10 \%$ buffered formalin then embedded in paraffin. Paraffin-embedded tissues were sliced into $5 \mu \mathrm{m}$ thick sections then used for H\&E or immunohistochemical staining. For immunocytochemistry, cells were seeded on glass coverslips at a density of $2.0 \times 10^{4} / \mathrm{cm}^{2}$ then cultured to about $60 \%$ confluence prior to fixation and permeabilization in $10 \%$ buffered formalin and $0.5 \%$ Triton X-100. Cells were stained using the SP-9000 Histostain ${ }^{\mathrm{TM}}$-Plus Kits (BIOZ, Porter Drive Palo Alto, CA, USA) according to the manufacturer's instructions using antibodies against PRC1 (1:150, Abcam, Cambridge, UK) and Ki-67 (1:200, ImmunoWay, Plano, TX, USA) were. Specific antigens in ten randomly-selected fields were visualized by two independent investigators using a light microscope at a power of $\times 400$, as per the method of Kreisberg et al. ${ }^{49}$

\section{p53 interference}

Cells of $1 \times 10^{4}$ per well were seeded into the 6-well plates and incubated at $37{ }^{\circ} \mathrm{C}$ for $24 \mathrm{~h}$. Then, $0.1 \%$ Nutlin-3 (SigmaAldrich, St Louis, MO, USA) dissolved in dimethyl sulfoxide (DMSO) (MP Biomedicals, Santa Ana, CA, USA) to a concentration of $25 \mu \mathrm{M}$ for Cal-27 and $20 \mu \mathrm{M}$ for HSC-2 was added into the cells respectively. Identically, $0.1 \%$ Pifithrin- $\alpha$ (Beyotime, Shanghai, China) dissolved in DMSO to a concentration of $35 \mu \mathrm{M}$ for Cal-27 and HSC-2 cells was added. After $48 \mathrm{~h}$, the total protein was extracted for western blot.

\section{Quantitative real-time PCR (qRT-PCR)}

For gene-detection, total RNA was isolated from cells using Trizol reagent (Invitrogen, Grand Island, NY, USA) and quantified by measuring the absorbance at $260 \mathrm{~nm}$. First-strand cDNA was synthesized from total RNA using a Perfect Real Time PrimeScript ${ }^{\circledR}$ RT reagent kit (Takara, Shiga, Japan), according to the manufacturer's protocol. The cDNA was quantified by polymerase chain reaction (PCR) on a 7300HT Real-Time PCR System using SYBR ${ }^{\circledR}$ Premix Ex TaqTM II (Takara, Shiga, Japan). GAPDH was used as the endogenous control. The PCR primer pairs were synthesized as follows: PRC1 sense: 5'-TAGACCACACCCCAGACACAAG-3' and antisense: $5^{\prime}$-CCCCTC ACACACTGCTTCATT-3'; GAPDH sense: 5'-CTTT GGTATCGTGGAAGGACTC-3' and antisense: $5^{\prime}$-GTA
GAGGCAGGGATGATGTTCT-3'. All the procedures were performed according to the manufacturer's instructions. Additionally, the comparative CT method ( $\triangle \Delta C T$ method) was used to quantify target gene expression in comparison with the control.

\section{Western blot analysis}

Whole-cell protein extracts were prepared using a lysis buffer containing phosphatase inhibitor, protease inhibitor and pheylmethylsulfonyl fluoride (KeyGEN BioTECH, Jiangsu, China). For western blot analysis, proteins were subjected to 10 and 5\% SDS-PAGE (Beyotime, Shanghai, China) using standard techniques. After transferring to the PVDF membrane (Millipore, Darmstadt, Germany), the protein contents were probed with antiEGFR, TßRII, AURKA and p53, pho-p53 $\left(\mathrm{Ser}^{392}\right)$ (1:500, ImmunoWay, Plano, TX, USA), and anti-PRC1 (1:5000, Abcam, Cambridge, UK) antibodies, using GAPDH as a control. Horseradish peroxidase-conjugated secondary antibodies were reacted with the primary antibodies above. Proteins were visualized using an electrochemiluminescence system (Pierce Biotech, Grand Island, NY, USA).

\section{Lentiviral construction and cell transfection}

Recombinant lentivirus vectors were purchased from Shanghai Genechem Co., Ltd (http://www.genechem. com.cn). Targeted sequences for shRNA PRC1 (shPRC1) were designed as follows: (1). PRC1-RNAi (22440-1): $5^{\prime}$ GGAACATTCAAAGGCATTT-3'; (2). PRC1-RNAi (22442-1): 5'-GCTGCAATTAGAAGTGGAT-3'; (3). PRC1-RNAi (22443-1): 5'-AGCTTTGTTAAATTGTGTT-3'. Recombinant viruses were generated using 293T packaging cells and a U6-MCS-UbiEGFP vector. Specific and control viruses were denoted si$\mathrm{PRC} 1$ and negative control lentivirus (si-NC), respectively. Subsequently, si-PRC1 and si-NC were transfected into HSC-2 and Cal-27 cells and cultured in six-well plates for $12 \mathrm{~h}$. The supernatant was removed and replaced with fresh culture medium containing $10 \%$ FBS. The cells were harvested for qRT-PCR and western blot analysis 72-96 h after transfection. When the infected cells exhibited a mean $80 \%$ decrease in PRC1 expression, they were used in the following experiments.

\section{Cell cycle analysis}

The distribution of cells in the cell cycle was evaluated by FCM. After seeding the cells at a density of $5.0 \times 10^{6}$ cells/petri dish (100 mm diameter), they were incubated in DMEM without serum for $24 \mathrm{~h}$ and then replaced by DMEM containing $10 \%$ FBS for $72 \mathrm{~h}$. The cells were then trypsinized, resuspended in $70 \%$ ethanol, washed and incubated in phosphate-buffered saline (PBS) containing PI and RNAse A (KeyGEN Bio-TECH, Jiangsu, China) for 
$30 \mathrm{~min}$ at $37^{\circ} \mathrm{C}$. DNA contents were then analyzed using an Elite ESP flow cytometry (Beckman Coulter, CA, USA).

\section{Cell proliferation assay}

A cell counting kit-8 (CCK-8, Dojindo, Japan) assay was used to quantify cell metabolism by measuring optical density (OD) of HSC-2 and Cal-27 cells after treatment in si-PRC1, si-NC and blank. Cells were firstly cultivated in a 96-well plate at a concentration of $1.0 \times 10^{3}$ per well, and then incubated at $37^{\circ} \mathrm{C}$ for $24,48,72$ and $96 \mathrm{~h}$. For each time point, the $\mathrm{OD}$ at $450 \mathrm{~nm}$ was measured using a spectrophotometer (Thermo, Grand Island, NY, USA) $2 \mathrm{~h}$ after adding CCK-8, according to the manufacturer's instructions. All tests were performed in triplicate.

\section{Measurement of cell death}

HSC-2 and Cal-27 cells were transfected with either siPRC1 or si-NC, respectively. After $72 \mathrm{~h}$ post-transfection, detached cells were collected from the medium by centrifugation $\left(300 \times g, 4^{\circ} \mathrm{C}, 5 \mathrm{~min}\right)$ while those that were adherent were harvested by trypsinization without ethylenediaminetetraacetic acid. For the apoptosis assay, $1.0 \times 10^{5}$ cells were resuspended in binding buffer $(100$ $\mu$ l) containing $5 \mu \mathrm{l}$ Annexin V-Alexa Fluor 647 and $10 \mu \mathrm{l}$ PI for $15 \mathrm{~min}$ in the dark at room temperature. After adding $400 \mu \mathrm{l}$ PBS, cell apoptosis was detected using an Elite ESP flow cytometer (Beckman Coulter, CA, USA). Annexin V-positive cells were considered apoptotic. Trypan blue (Beyotime, Shanghai, China) and PI/calcein fluorescence staining (KeyGEN Bio-TECH, Jiangsu, China) were performed according to the manufacturer's instructions.

\section{Tumorigenesis assay in nude mice}

All experiments involving animals were conducted in accordance with the U.S. Public Health Service's policy on the humane care and use of laboratory animals. Eighteen 4-week-old BALB/c athymic nude mice were used, half being males and half females, purchased from the State Key Laboratory of Biotherapy and Cancer Center at Sichuan University. They were randomly divided into the three groups: negative control, si-PRC1 or si-NC. PRC1 mimic-transfected HSC-2 cells $(5.0 \times$ $10^{5}$ ) were suspended in $0.1 \mathrm{ml} \mathrm{PBS}$ and then injected subcutaneously into the dorsal of nude mice. Tumor major and minor axes of each tumor were measured every 5 days using calipers from day 5 following injection. Tumor volume was calculated as follows: tumor volume $=0.52 \times($ major axis $) \times(\text { minor axis })^{250}$. On the 25th day after injection, all mice were killed using luxation and the tumors were excised and weighed. The expression of $\mathrm{Ki}-67$ and PRC1 in tumor samples was mainly detected by IHC.

\section{Acknowledgements}

This study was supported by the National Nature Science Foundation of China (Nos. 81772898, 81272962), the Key Research and Development Program of Sichuan Province (No. 2017SZ0104) and the Fundamental Research Funds for the Central Universities (No. 2012017yjsy119).

\section{Author details}

${ }^{1}$ State Key Laboratory of Oral Diseases, Department of Oral Medicine, West China Hospital of Stomatology, Sichuan University, 610041 Chengdu, Sichuan, China. ${ }^{2}$ Department of Preventive Dentistry, West China Hospital of Stomatology, Sichuan University, 610041 Chengdu, Sichuan, China. ${ }^{3}$ State Key Laboratory of Oral Diseases, West China Hospital of Stomatology, Sichuan University, 610041 Chengdu, Sichuan, China. ${ }^{4}$ Department of Pediatric Dentistry, West China Hospital of Stomatology, Sichuan University, 610041 Chengdu, Sichuan, China. ${ }^{5}$ Department of Stomatology, The Affiliated Hospital of Qingdao University, 266003 Qingdao, Shandong, China

\section{Authors' contributions}

H.Z., Y.G., and W.G. proposed the experimental hypothesis and design; Y.G., X.S., F.W., R.Z., and D.L. performed experiments in vitro; Y.G., C.W., F.W., R.Z., and Y.T. involved in animal studies; F.W., X.S., and R.Z. performed statistical analysis; X.L., Y.L., and X.K. provided advice on the policy perspective for this research; F.W. X.S., and R.Z. wrote the manuscript; H.Z., Y.G., and W.G. revised the manuscript. All authors read and approved the final manuscript.

Conflict of interest

The authors declare that they have no conflict of interest.

\section{Publisher's note}

Springer Nature remains neutral with regard to jurisdictional claims in published maps and institutional affiliations.

Supplementary Information accompanies this paper at (https://doi.org/ 10.1038/s41419-018-0618-6).

Received: 22 October 2017 Accepted: 24 April 2018

Published online: 11 May 2018

\section{References}

1. Chaturvedi, A. K. et al. Worldwide trends in incidence rates for oral cavity and oropharyngeal cancers. J. Clin. Oncol. 31, 4550-4559 (2013).

2. Fakhry, C. et al. The prognostic role of sex, race, and human papillomavirus in oropharyngeal and nonoropharyngeal head and neck squamous cell cancer. Cancer 123, 1566-1575 (2017)

3. Speight, P. M. et al. Screening for oral cancer-a perspective from the Global Oral Cancer Forum. Oral. Surg. Oral. Med. Oral. Pathol. Oral. Radiol. 123, 680-687 (2017).

4. Torre, L. A. et al. Global cancer statistics, 2012. Ca Cancer J. Clin. 65, 87-108 (2015).

5. Bloebaum, M., Poort, L., Bockmann, R. \& Kessler, P. Survival after curative surgical treatment for primary oral squamous cell carcinoma. J. Craniomaxillofac. Surg. 42, 1572-1576 (2014).

6. Dik, E. A. et al. Resection of early oral squamous cell carcinoma with positive or close margins: relevance of adjuvant treatment in relation to local recurrence: margins of $3 \mathrm{~mm}$ as safe as $5 \mathrm{~mm}$. Oral. Oncol. 50, 611-615 (2014).

7. Shah, J. P. \& Gil, Z. Current concepts in management of oral cancer surgery. Oral. Oncol. 45, 394-401 (2009).

8. Le, T. C. et al. Molecularly targeted therapy based on tumour molecular profiling versus conventional therapy for advanced cancer (SHIVA): a multicentre, open-label, proof-of-concept, randomised, controlled phase 2 trial. Lancet Oncol. 16, 1324-1334 (2015).

9. Kalia, M. Personalized oncology: recent advances and future challenges. Metabolism 62, S11-14 (2013).

10. Sekulic, A. et al. Efficacy and safety of vismodegib in advanced basal-cell carcinoma. N. Engl. J. Med. 366, 2171-2179 (2012). 
11. Allen, W. L. et al. A systems biology approach identifies SART1 as a novel determinant of both 5-fluorouracil and SN38 drug resistance in colorectal cancer. Mol. Cancer Ther. 11, 119-131 (2012).

12. Meng, W. et al. A systems biology approach identifies effective tumor-stroma common targets for oral squamous cell carcinoma. Cancer Res. 74, 2306-2315 (2014).

13. Wang, E., Lenferink, A. \& O'Connor-McCourt, M. Cancer systems biology: exploring cancer-associated genes on cellular networks. Cell Mol. Life Sci. 64 1752-1762 (2007)

14. Subramanian, R., Ti, S. C., Tan, L., Darst, S. A. \& Kapoor, T. M. Marking and measuring single microtubules by PRC1 and kinesin-4. Cell 154, 377-390 (2013).

15. Roostalu, J., Schiebel, E. \& Khmelinskii, A. Cell cycle control of spindle elongation. Cell Cycle 9, 1084-1090 (2010).

16. Subramanian, R. et al. Insights into antiparallel microtubule crosslinking by PRC1, a conserved nonmotor microtubule binding protein. Cell 142, 433-443 (2010).

17. Kanehira, M. et al. Oncogenic role of MPHOSPH1, a cancer-testis antigen specific to human bladder cancer. Cancer Res. 67, 3276-3285 (2007).

18. Shimo, A. et al. Elevated expression of protein regulator of cytokinesis 1, involved in the growth of breast cancer cells. Cancer Sci. 98, 174-181 (2007)

19. Gruneberg, U. et al. KIF14 and citron kinase act together to promote efficient cytokinesis. J. Cell Biol. 172, 363-372 (2006).

20. Li, C., Lin, M. \& Liu, J. Identification of PRC1 as the p53 target gene uncovers a novel function of p53 in the regulation of cytokinesis. Oncogene 23, 9336-9347 (2004)

21. Liu, J. et al. PRC1 cooperates with CLASP1 to organize central spindle plasticity in mitosis. J. Biol. Chem. 284, 23059-23071 (2009).

22. Pal, D., Wu, D., Haruta, A., Matsumura, F. \& Wei, Q. Role of a novel coiled-coi domain-containing protein CCDC69 in regulating central spindle assembly. Cell Cycle 9, 4117-4129 (2010).

23. Duellberg, C., Fourniol, F. J., Maurer, S. P., Roostalu, J. \& Surrey, T. End-binding proteins and Ase1/PRC1 define local functionality of structurally distinct parts of the microtubule cytoskeleton. Trends Cell Biol. 23, 54-63 (2013).

24. Sasabe, M. \& Machida, Y. Regulation of organization and function of microtubules by the mitogen-activated protein kinase cascade during plant cytokinesis. Cytoskeleton 69, 913-918 (2012).

25. Yun, H. J. et al. Transcriptional targeting of gene expression in breast cancer by the promoters of protein regulator of cytokinesis 1 and ribonuclease reductase 2. Exp. Mol. Med. 40, 345-353 (2008)

26. Mollinari, C. et al. Ablation of PRC1 by small interfering RNA demonstrates that cytokinetic abscission requires a central spindle bundle in mammalian cells, whereas completion of furrowing does not. Mol. Biol. Cell 16, 1043-1055 (2005).

27. Shrestha, S., Wilmeth, L. J., Eyer, J. \& Shuster, C. B. PRC1 controls spindle polarization and recruitment of cytokinetic factors during monopolar cytokinesis. Mol. Biol. Cell 23, 1196-1207 (2012).

28. Zheng, J. et al. Apoptosis-related protein-1 acts as a tumor suppressor in cholangiocarcinoma cells by inducing cell cycle arrest via downregulation of cyclin-dependent kinase subunits. Oncol. Rep. 35, 809-816 (2016).

29. Zhan, P. et al. Protein regulator of cytokinesis-1 expression: prognostic value in lung squamous cell carcinoma patients. J. Thorac. Dis. 9, 2054-2060 (2017).

30. Zhan, P. et al. PRC1 contributes to tumorigenesis of lung adenocarcinoma in association with the Wnt/beta-catenin signaling pathway. Mol. Cancer 16, 108 (2017).

31. Ray, R. B., Raychoudhuri, A., Steele, R. \& Nerurkar, P. Bitter melon (Momordica charantia) extract inhibits breast cancer cell proliferation by modulating cell cycle regulatory genes and promotes apoptosis. Cancer Res. 70, 1925-1931 (2010).

32. Paruthiyil, S. et al. Estrogen receptor beta inhibits human breast cancer cell proliferation and tumor formation by causing a G2 cell cycle arrest. Cancer Res. 64, 423-428 (2004).

33. Barzegar, E. et al. Effects of berberine on proliferation, cell cycle distribution and apoptosis of human breast cancer T47D and MCF7 cell lines. Iran. J. Basic Med. Sci. 18, 334-342 (2015).

34. Zhang, B. et al. Elevated PRC1 in gastric carcinoma exerts oncogenic function and is targeted by piperlongumine in a p53-dependent manner. J. Cell Mol. Med. 21, 1329-1341 (2017).

35. Team, B. et al. Ki-67 can be used for further classification of triple negative breast cancer into two subtypes with different response and prognosis. Breast Cancer Res. 13, R22 (2011).

36. Ezziddin, S. et al. Impact of the $\mathrm{Ki}-67$ proliferation index on response to peptide receptor radionuclide therapy. Eur. J. Nucl. Med. Mol. Imaging 38, 459-466 (2011).

37. Dwivedi, N., Chandra, S., Kashyap, B., Raj, V. \& Agarwal, A. Suprabasal expression of Ki-67 as a marker for the severity of oral epithelial dysplasia and oral squamous cell carcinoma. Contem. Clin. Dent. 4, 7 (2013).

38. Alsina-Sanchis, E. et al. The TGFbeta pathway stimulates ovarian cancer cell proliferation by increasing IGF1R levels. Int. J. Cancer 139, 1894-1903 (2016).

39. Jin, Y. et al. EGFR/Ras signaling controls drosophila intestinal stem cell proliferation via Capicua-regulated genes. PLoS Genet. 11, e1005634 (2015).

40. Premkumar, D. R., Jane, E. P. \& Pollack, I. F. Cucurbitacin-l inhibits Aurora kinase A, Aurora kinase B and survivin, induces defects in cell cycle progression and promotes ABT-737-induced cell death in a caspase-independent manner in malignant human glioma cells. Cancer Biol. Ther. 16, 233-243 (2015).

41. Song, W. et al. Kaempferol induces cell cycle arrest and apoptosis in renal cell carcinoma through EGFR/p38 signaling. Oncol. Rep. 31, 1350-1356 (2014).

42. Meng, W. et al. Downregulation of TGF-beta receptor types II and III in oral squamous cell carcinoma and oral carcinoma-associated fibroblasts. BMC Cancer 11, 88 (2011).

43. Vaughan, C. A. et al. Addiction of lung cancer cells to GOF p53 is promoted by up-regulation of epidermal growth factor receptor through multiple contacts with p53 transactivation domain and promoter. Oncotarget 7, 12426-12446 (2016).

44. Wang, W., Cheng, B., Miao, L., Mei, Y. \& Wu, M. Mutant p53-R273H gains new function in sustained activation of EGFR signaling via suppressing miR-27a expression. Cell Death Dis. 4, e574 (2013).

45. Normanno, N. et al. Epidermal growth factor receptor (EGFR) signaling in cancer. Gene 1, 2-16 (2006).

46. D'Ambrosio, S. M., Han, C., Pan, L., Kinghorn, A. D. \& Ding, H. Aliphatic acetogenin constituents of avocado fruits inhibit human oral cancer cell proliferation by targeting the EGFR/RAS/RAF/MEK/ERK1/2 pathway. Biochem. Biophys. Res. Commun. 3, 465-469 (2011).

47. Yamada, T. et al. PTHrP promotes malignancy of human oral cancer cell downstream of the EGFR signaling. Biochem. Biophys. Res. Commun. 3, 575-581 (2008).

48. Pan, C. et al. Aurora kinase small molecule inhibitor destroys mitotic spindle, suppresses cell growth, and induces apoptosis in oral squamous cancer cells. Oral. Oncol. 44, 639-645 (2008).

49. Kreisberg, J. I. et al. Phosphorylation of Akt (Ser473) is an excellent predictor of poor clinical outcome in prostate cancer. Cancer Res. 64, 5232-5236 (2004).

50. Li, Q. et al. A central role for mTOR kinase in homeostatic proliferation induced CD8 ${ }^{+}$T cell memory and tumor immunity. Immunity 34, 541-553 (2011). 